Journal of Engineering and Applied Sciences 15 (1): 48-59, 2020

ISSN: 1816-949X

(C) Medwell Journals, 2020

\title{
Performance of Differential CSK under Color Noise: A Comparison with CSK
}

\author{
Raghad I. Hussein, Zahir M. Hussain and Salah A. Albermany \\ Faculty of Computer Science and Mathematics, University of Kufa, Najaf, Iraq
}

\begin{abstract}
In this research, we present a comprehensive study on the performance of chaotic communication systems under Gaussian and color noise. We selected the most useful systems for short range communications: Chaos Shift Key (CSK) and Differential Chaos Shift Key (DCSK) systems. Logistic map and tent map have been used to generate Chaos in these systems. Color noise has been generated by filtering Gaussian noise via. an FIR digital filter. Various spreading factors have been used. Although, DCSK outperforms CSK in complexity, it is shown that CSK outperforms DCSK under Gaussian and color noise and various spreading Factors (SF) in terms of Bit Error Rate (BER). In addition, it is shown that CSK is more secure than DCSK. both systems have been simulated using MATLAB R2018a.
\end{abstract}

Key words: CSK, DCSK, chaotic map, tent map, logistic map, Gaussian noise, AWGN, color noise, CGN

\section{INTRODUCTION}

Chaos is a word derived from the ancient Greek which means unpredictable behavior and the opposite of the cosmos or order. However in Chaos theory, the term "Chaos" is not an antithesis of the cosmos or absence of order but in fact has a very subtle order in itself not quite obvious as ordered systems. The history of Chaos in the scientific community has to be stretched back to the time when Newton solved the two body problem in the universe using his newly invented differential equation (Gleick, 1987; Rupak, 2011; Strogatz, 1994). Chaos theory is a blanketing theory that covers all aspects of science, hence, it appears everywhere in the world today Mathematics, Physics, Biology, Finance, Communication, Computer and even music. The term complexity theory and complex systems theory provide a better description of the subject matter but the term Chaos theory will be used throughout this proposal as it is more widely accepted. Chaotic signals in the time domain are neither periodic nor quasi-periodic and are unpredictable in the long term. This unpredictable phenomenon manifests itself as a wideband noise-like power spectrum in the frequency domain. The chaotic dynamic system can be classified into continuous-time and discrete-time (Luke, 2012). Chaos-based communication systems have been shown to provide certain advantages over conventional communication systems. However, there are still plentiful of issues to be resolved before chaos-based systems can be put into practical use. Hence, a study on the use of Chaos Shift Key (CSK) to improve Chaos communication makes it possible for further practical improvement. More research is needed in this area and there is a plenty of room for further study and improvement. A chaos-based communications system could also improve privacy, security and probability of intercept in as much as chaotic sequences, unlike pseudorandom sequences can be made completely non periodic (Larson et al., 2006).

Chaos-based communication systems could also improve privacy, security and the probability of interception because chaotic sequences, unlike pseudorandom sequences can be made completely aperiodic (Larson et al., 2006). We may classify chaos-based communication systems into two main categories, namely, coherent and non-coherent systems (Kaddoum, 2016). In coherent systems, the receiver needs to reproduce the chaotic carrier for demodulation whereas in the non-coherent system, demodulation is performed solely based on the received signal (Lau and Tse, 2003).

In this study, we focus on Chaos Shift Keying (CSK) and (DCSK) which is one method of chaotic digital modulation in discrete-time. An efficient Chaos generator that can be used to generate (CSK and DCSK) sequences is logistic map and tent map (Lau and Hussain, 2005) where different sequences can be generated using the same generator but with different initial conditions. We used a chaotic logistic map and the tent map for the generation of chaotic sequences tent map which is another commonly used discrete chaotic map, Fig. 1 (Lau and Hussain, 2005 Lau et al., 2004). The chaotic sequence generated by the tent map has been widely applied in the field of chaotic spectrum communication, encryption system chaotic optimum algorithm and so on (Sun, 2016). To enhance the security and noise performance, a larger spreading factor can be used.

We can measure the performance of the CSK system generated using the LCG1 map in different noisy environments with different SFs. The following results show the BER for SNRs in the range (Elmirghani, 1995). The BER is used to measure the effect of channel imperfections on the transmitted signal that is obtained by

Corresponding Author: Zahir M. Hussain , Faculty of Computer Science and Mathematics, University of Kufa, Najaf, Iraq 


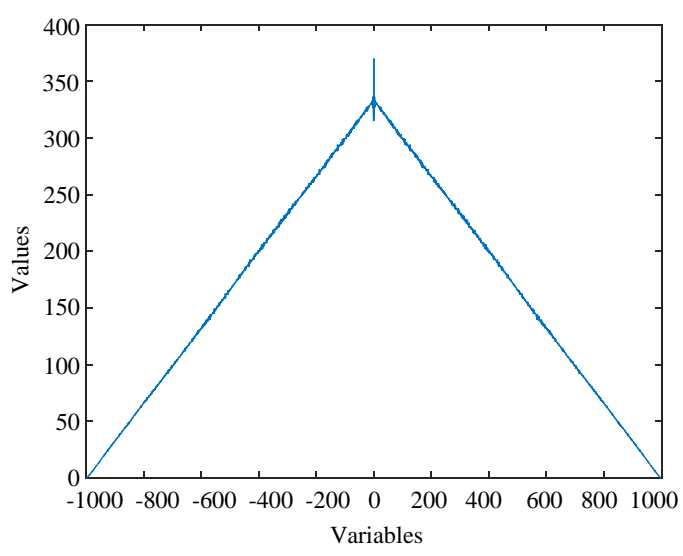

Fig. 1: Autocorrelation function of tent map (Chaos Rzz; tent map)

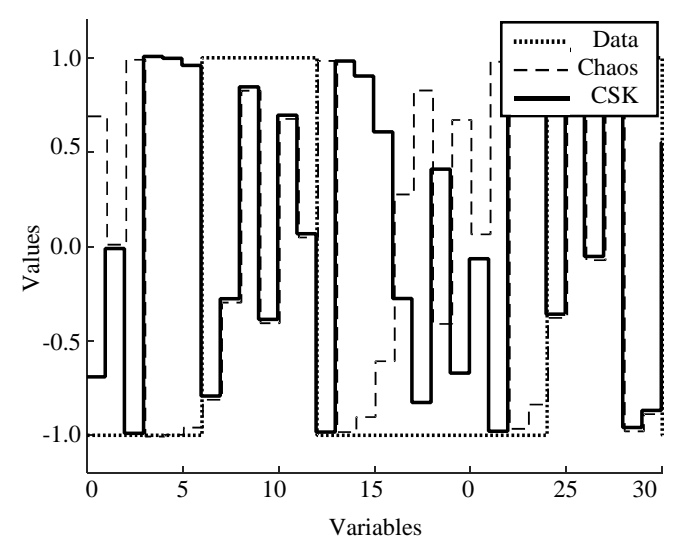

Fig. 2: CSK signal under logistic map

counting the number of incorrectly received bits and dividing this number by the total number of bits transmitted (Larson et al., 2006). We illustrate in Fig. 2 CSK generated by an LCG map using Eq. 4 that was used to generate a chaotic signal with a Spreading Factor $\mathrm{SF}=3$. Note that Chaos is ranging from -1 to +1 .

\section{MATERIALS AND METHODS}

Chaos Shift Keying (CSK): In recent years, Chaos Shift Keying (CSK) has proved to offer a potential advantage over conventional methods due to its simplicity and high level of unpredictability. Chaos Shift Keying (CSK) was first proposed in as show in Fig. 3 (Dedieu et al., 1993). Where in binary Chaos shift keying modulation, chaotic signals carrying different bit energies are used to transmit the binary information (Lau and Hussain, 2005; Lau et al., 2004; Lau and Tse, 2003; Kolumban, 2000; Kolumban et al., 2002; Abel and Schwarz, 2002; Lau, 2006). An information signal is encoded by transmitting one chaotic signal $g_{1}(t)$ or $g_{0}(t)$ at a time. For example, if the information signal binary bit " 1 " occurs at time $t$, the chaos signal $\mathrm{g}_{1}(\mathrm{t})$ is to be sent and for information bit " 0 ", the chaos signal $\mathrm{g}_{0}(\mathrm{t})$ is to be sent. The block diagram of a (binary) CSK communication system is depicted in Fig. 3. As can be observed, only one chaos generator is used at the transmitter terminal. In such a system, i-th bit $\alpha_{i} \in\{+1,-1\}$ is represented by one chaotic sequence $g_{k}=\left\{g_{k, i}\right\}$ where, $k=1,2, \ldots, i=1,2, \ldots, g_{k, i}$ is the $\mathrm{k}$-th component chaotic sample with expected value $\varepsilon$ $\left(\mathrm{g}_{\mathrm{k}, \mathrm{i}}\right)=0$. Assume that the durations of a chaotic sample and a bit denote by $\mathrm{T}_{\mathrm{c}}$ and $\mathrm{T}_{\mathrm{b}}$, respectively and the global spreading factor is denoted by:

$$
2 \beta=\mathrm{T}_{\mathrm{b}} / \mathrm{T}_{\mathrm{c}}
$$

Accordingly, the i-th modulated sample output from a CSK modulator corresponding to $\alpha_{\mathrm{i}}$ is expressed as $\mathrm{s}=\alpha_{i} g_{k}$ where $\mathrm{i}=1,2, \ldots, 2 \beta, \mathrm{g}_{\mathrm{k}, \mathrm{i}}$ serves as the carrier and $s_{k}=\left[s_{k, 1}, s_{k, 2}, \ldots, s_{k, 2 \beta}\right]$ denotes the overall baseband transmitted signal during the $\mathrm{k}$-th bit duration. Note that both $s_{k, i}$ and $g_{k, i}$ are real numbers. This signal is then passed through a noisy channel and detected by a coherent demodulator. Based on the received signal vector $r_{k}=\left[r_{k, 1}, r_{k, 2}, \ldots, r_{k, 2 \beta}\right]$ which represents the received CSK in the $\mathrm{k}$-th transmission period (i.e., bit duration), a decision metric (demodulator output) $y_{i}$ is computed using:

$$
y_{i}=\sum_{k=2 \beta(i-1)+1}^{2 \beta} r_{k} g_{k}
$$

Then the decoded bit is determined based on a hard-decision rule: $\bar{\alpha}_{\mathrm{i}}=+1$ if $\mathrm{y}_{\mathrm{i}} \geq 0$ and $\widehat{\alpha}_{\mathrm{i}}=-1$ otherwise. As can be seen from Fig. 3, the synchronization circuit plays a crucial role in the detection process because it is required to recover the "noise-like" chaotic carrier " $g_{k}$ based on the noisy received signal $r_{k}$. When the noise is extremely small and the channel varies slowly, synchronization of chaotic circuits and chaotic maps can be achieved using adaptive controllers or neural networks. Unfortunately, the existing chaos synchronization algorithms are not able to provide satisfactory performance in noisy environments and thus, the realization of synchronization circuit is still an intractable problem at present. The two chaotic signals can come from two different chaos systems or the same system with different parameters. The transmitted signal is given by:

$$
s(t)=\left\{\begin{array}{c}
g_{0}(t), \text { symbol"0" is transmitted } \\
g_{1}(t), \text { symbol"1"is transmitted }
\end{array}\right.
$$

Differential Chaos Shift Keying (DCSK): The differential Chaos shift keying was introduced by Kolumban et al. (1996), Cai et al. (2017) and shown to outperform CSK schemes when the channel condition is so poor that it is impossible to achieve chaotic 


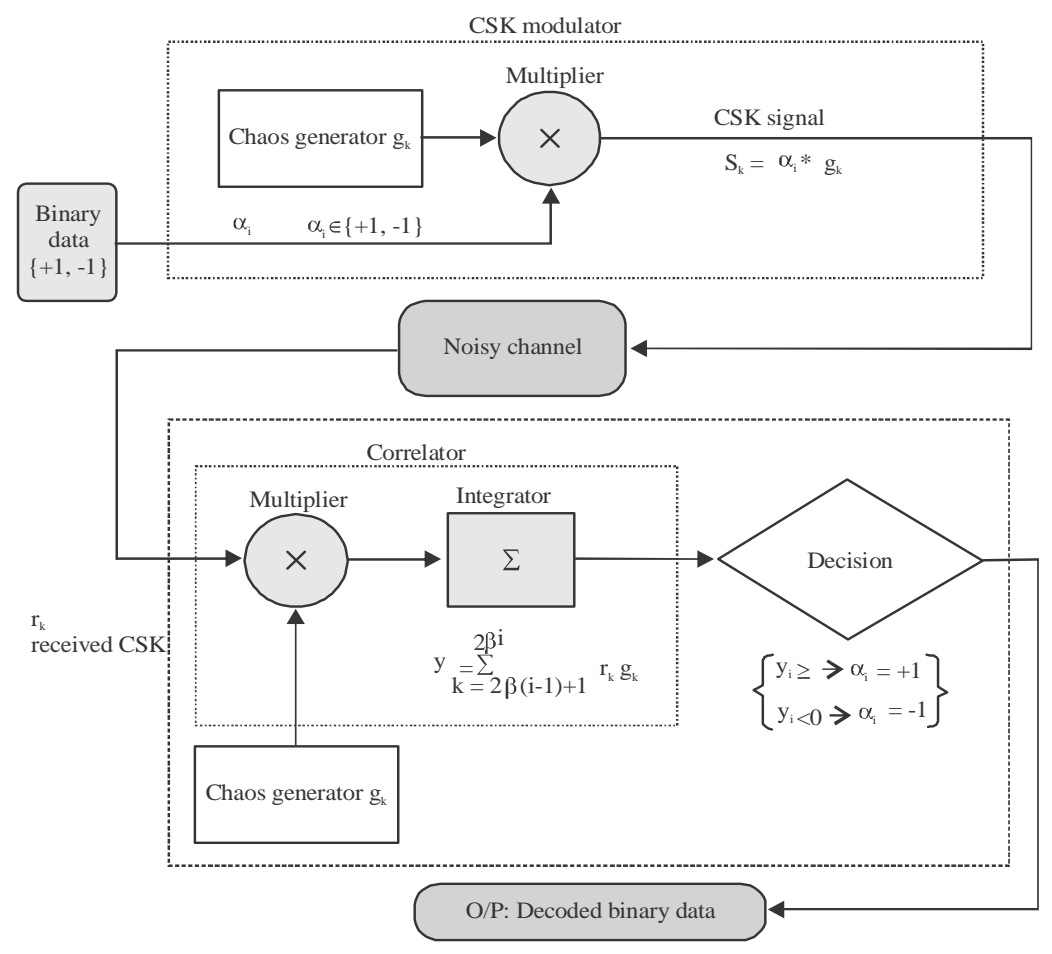

Fig. 3: Chaos Shift Keying (CSK) system

synchronization as shown in Fig. 4. This modulation scheme is similar to that of the Differential Phase Shift Keying (DPSK) except that the transmitted signal is a chaotic-generated signal. In DCSK modulation, each transmitted symbol duration is divided into two identical time slots. The first time slot serves as a reference while the second slot carries the information. If bit " 1 " is to be sent, the chaotic reference signal (in the first slot) is repeated in the second slot, if bit " 0 " is to be sent an inverted copy of the reference signal (in the first slot) will be sent. In such a system, $i$-th bit $\alpha_{i} \in\{+1,-1\}$ is represented by one chaotic sequence $\mathrm{g}_{\mathrm{k}}=\left\{\mathrm{g}_{\mathrm{k}, \mathrm{i}}\right\}$ where $\mathrm{k}=1,2, . ., \mathrm{i}=1,2, \ldots, \mathrm{g}_{\mathrm{k}}$ is the $\mathrm{k}$-th component chaotic sample ( $g_{k}$ chaos generator) and $\varepsilon\left\{g_{k, i}\right\}=0$. Assume that the durations of a chaotic sample and a bit denote by $T_{c}$ and $T_{b}$, respectively and the global spreading factor is denoted by:

$$
2 \beta=\mathrm{T}_{\mathrm{b}} / \mathrm{T}_{\mathrm{c}}
$$

Accordingly, the k-th modulated sample output from a DCSK modulator corresponding to $\alpha_{\mathrm{i}}$ is expressed as follows if the transmitted signal for an information bit "1":

$$
\text { DCSK signal }: s_{k}\left\{\begin{array}{c}
g_{k} \text { Fort }_{i, 0} \leq t \leq t_{i, 0}+\beta \\
\alpha_{i} g_{k-\beta} \text { Fort }_{i, 0}+\beta+1 \leq t \leq \beta_{i}
\end{array}\right.
$$

If the transmitted signal for an information bit " 0 " we have:

$$
\mathrm{s}_{\mathrm{k}}\left\{\begin{array}{c}
\mathrm{g}_{\mathrm{k}} \text { Fort }_{\mathrm{i}, 0} \leq \mathrm{t} \leq \mathrm{t}_{\mathrm{i}, 0}+\beta \\
-\alpha_{\mathrm{i}} \mathrm{g}_{\mathrm{k}-\mathrm{\beta}} \text { Fort }_{\mathrm{i}, 0}+\beta+1 \leq \mathrm{t} \leq \beta_{\mathrm{i}}
\end{array}\right.
$$

where, $t_{i, 0}=2 \beta(i-1)+1$. The system switch at $t=\beta T_{c}$. Note that $g_{k, i}$ serves as the carrier and that the vector $s_{k}=\left[s_{k, 1}, s_{k, 2}, \ldots, s_{k, 2 \beta}\right]$ denotes the overall baseband transmitted signal during the k-th bit duration. Both $\mathrm{s}_{\mathrm{k}, \mathrm{i}}$ and $g_{k, i}$ are real numbers. This signal is then passed through a noisy channel, then detected by a coherent demodulator. Based on the received signal vector $r_{k}=\left[r_{k}\right.$, $\left.{ }_{1}, \mathrm{r}_{\mathrm{k}, 2}, \ldots, \mathrm{r}_{\mathrm{k}, 2 \beta}\right]$ which is the received DCSK in the $\mathrm{k}$-th transmission period (i.e., bit duration), a decision metric (demodulator output) $\gamma_{\mathrm{i}}$ is computed using

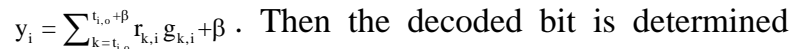
based on a hard-decision rule (threshold): $\widehat{\alpha}_{\mathrm{i}}=+1$ if $\mathrm{y}_{\mathrm{i}} \geq 0$ and $\widehat{\alpha}_{\mathrm{i}}=-1 \quad$ otherwise (Jawad et al., 2017).

Chaotic map: Chaos theory has been widely studied in the past a vast number of different forms of mathematical models are derived and investigated. Generations of chaotic maps came from many different directions. It can be a complex or simple control system, a mathematical equation such as differential equation or a simple circuit modeling like Chua circuit. In this research, we will concentrate on simple polynomial mapping which exhibits chaotic behavior that arises from simple non-linear 


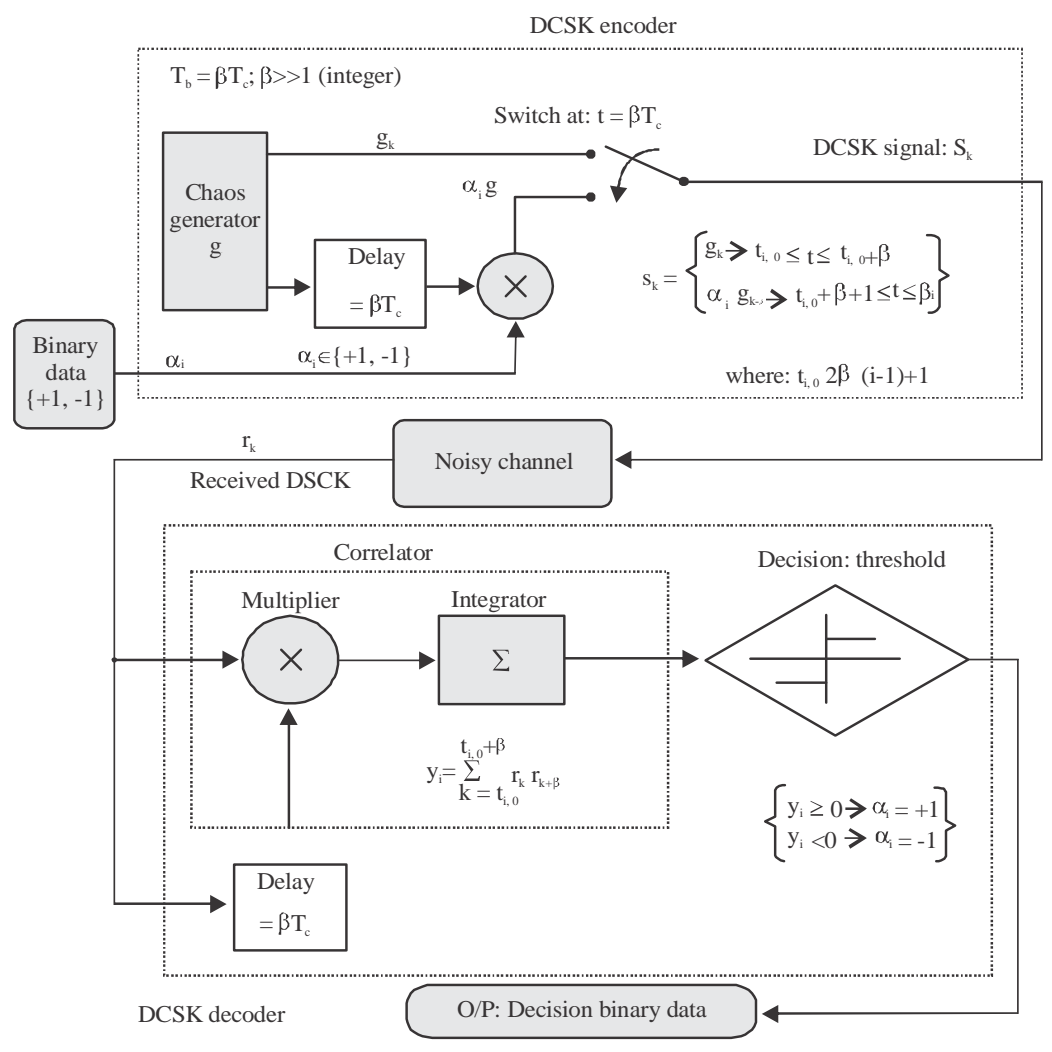

Fig. 4: Differential Chaos Shift Keying (DCSK)

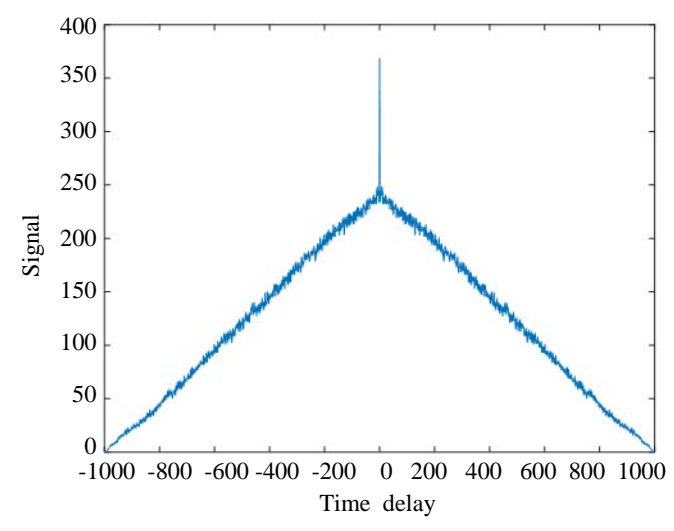

Fig. 5: Correlation functions of chaotic signals against time-delay vector, the generating system is logistic map (Choas Ryy; logistic map)

dynamical equation. Such a mathematical model of Chaos theory often involves repeated iteration of simple mathematical formulas. This type of mapping is called logistic map and tent map. Mathematically, it can be written as.

Logistic map: Logistic map is one of the simplest Chaos logistic maps used to generate chaotic signals. This map has been used in a number of applications including digital communications, Fig. 5 (Lau et al., 2004; Lau and Tse, 2003; Rao and Howard, 1996). Another dynamic system that is capable of exhibiting chaotic properties for spreading spectrum communication is proposed by Elmirghani (1995), Ushio (1994) in which:

$$
g_{n+1}=a \cdot g_{n}\left(1-g_{n}\right)
$$

where, $\alpha$ is the bifurcation parameter (or control) parameter which is considered to be in the interval of 3.57 $<\mathrm{a} \leq 4$ for a non-periodic Chaos system (Elmirghani, 1995; Ushio, 1994; Dedieu and Ogorzalek, 1997; Lian et al., 2001).

Tent map: It is a chaotic map defined as:

$$
x_{n+1}=\left\{\begin{array}{l}
\frac{x_{n}}{a}, x_{n} \in[0, a) \\
\frac{1-x_{n}}{1-a}, x_{n} \in[a, 1]
\end{array}\right.
$$

Tent map is piecewise linear because of this characteristic this makes the tent map easier to analyze than the logistic map. However, although, the form of the 


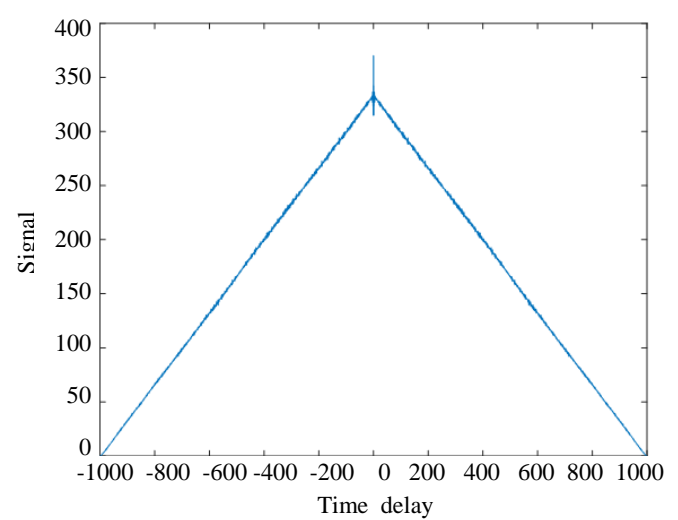

Fig. 6: Correlation functions of chaotic signals against time-delay vector, the generating system is tent map: (Choas Rzz; tent map)

tent map is simple and the equations are linear for certain parameter values the map can yield complex and chaotic behavior when the system parameter $\mathrm{a}$ is in $(0,1)$ in which case the variable $x_{n}$ would be in $(0,1)$. Tent map and logistic map are topological conjugates. When a varies on $(0,1)$, the system is chaotic. In particular, when $a=0.5$, the system is in a short-cycle state such as $(0.2,0.4,0.8)$. In this case, the system is simple and its complexity is small, Fig. 6. Therefore, for the application of the tent map system, we should choose reasownable system parameter and initial value (Sun, 2016).

Noisy channel: Channel refers to the path between transmitter and receiver whether it may be wired or wireless. Channel model refers to the mathematical modeling or mathematical equation describing the channel behavior between the transmitter and receiver. In our research the channel of communication effect with different noise that using to measure the performance of the systems in keeping the information signal or lose part of it (system resistance to external noise or system security). So, noise types that we use in this study are Gaussian and color noise as explained.

Gaussian noise: Gaussian noise is statistical noise having a Probability Density Function (PDF) equals to that of the normal distribution which is also known as the Gaussian distribution. In other words, the values that the noise can take on are Gaussian-distributed. The probability density function of a Gaussian random variable is given by (Hogg and Craig, 1978):

$$
\mathrm{p}(\mathrm{z})=\frac{1}{\sigma \sqrt{2} \pi} \mathrm{e}^{-(\mathrm{z}-\mathrm{m}) / 2 \sigma^{2}}
$$

Where:

$\mu$ : The mean value

$\sigma$ : The standard deviation of noise

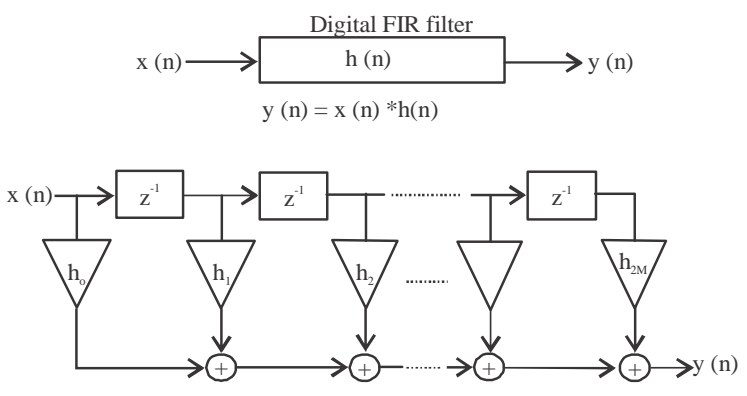

Fig. 7: FIR digital filter and its implementation

Color noise: The white noise has a flat power spectral density. Theoretically, it extends from zero frequency to very large frequencies tending to infinity. If one filter out white noise, it becomes colored, so, we obtain color noise $\mathrm{n}_{\mathrm{c}}(\mathrm{k})$ from Gaussian noise $\mathrm{n}(\mathrm{k})$ using an FIR filter with impulse response $\mathrm{h}(\mathrm{k})$ as follows:

$$
\mathrm{n}_{\mathrm{c}}(\mathrm{k})=\sum_{\mathrm{i}=0}^{\mathrm{N}} \mathrm{n}(\mathrm{i}) \mathrm{h}(\mathrm{k}-\mathrm{i})
$$

where, $\mathrm{N}$ is the filter order.

FIR digital filters: As a digital system, the function of the causal FIR filter is given by the following difference equation with a finite summation Fig. 7 (Hussain et al., 2011):

$$
\begin{aligned}
& \mathrm{y}(\mathrm{n})=\mathrm{h}(\mathrm{n}) * \chi(\mathrm{n})=\sum_{\mathrm{k}=0}^{\mathrm{N}-1} \mathrm{~h}(\mathrm{k}) \chi(\mathrm{n}-\mathrm{k})= \\
& \mathrm{h}_{0} \chi(\mathrm{n})+\mathrm{h}_{1} \chi(\mathrm{n}-1)^{+}, \ldots, \mathrm{h}_{\mathrm{N}-\mathrm{-}} \chi[(\mathrm{n}-(\mathrm{N}-1)]
\end{aligned}
$$

where, $h_{k}$ is used for $h(k)$ for simplicity. Taking the $\mathrm{z}$-transform of both sides we get:

$$
\mathrm{Y}(\mathrm{z})=\mathrm{h}_{0} \mathrm{X}(\mathrm{z})+\mathrm{h}_{1} \mathrm{z}^{-1} \mathrm{X}(\mathrm{z})+, \ldots, \mathrm{h}_{\mathrm{N}-\mathrm{1}} \mathrm{-}^{-(\mathrm{N}-1)} \mathrm{X}(\mathrm{z})
$$

Hence, the transfer function is given by:

$$
\mathrm{H}(\mathrm{z})=\frac{\mathrm{Y}(\mathrm{z})}{\mathrm{X}(\mathrm{z})}=\mathrm{h}_{0}+\mathrm{h}_{1} \mathrm{z}^{-1}+, \ldots,+\mathrm{h}_{\mathrm{N}-\mathrm{1}} \mathrm{-}^{-(\mathrm{N}-1)}
$$

Either from the difference equation or from the transfer function $\mathrm{H}(\mathrm{z})$ we can implement a causal FIR filter using delay elements and digital multipliers as in Fig. 7 (Hussain et al., 2011).

Linear phase FIR filters: A sufficient (but not necessary) condition that a FIR filter of impulse $h(n)$ with length $\mathrm{N}$ is a linear phase system is that $\mathrm{h}(\mathrm{n})$ is either (Hussain et al., 2011): Symmetric around the midpoint $\mathrm{n}_{0}=\mathrm{N}-1 / 2$, hence, the impulse response is characterized 

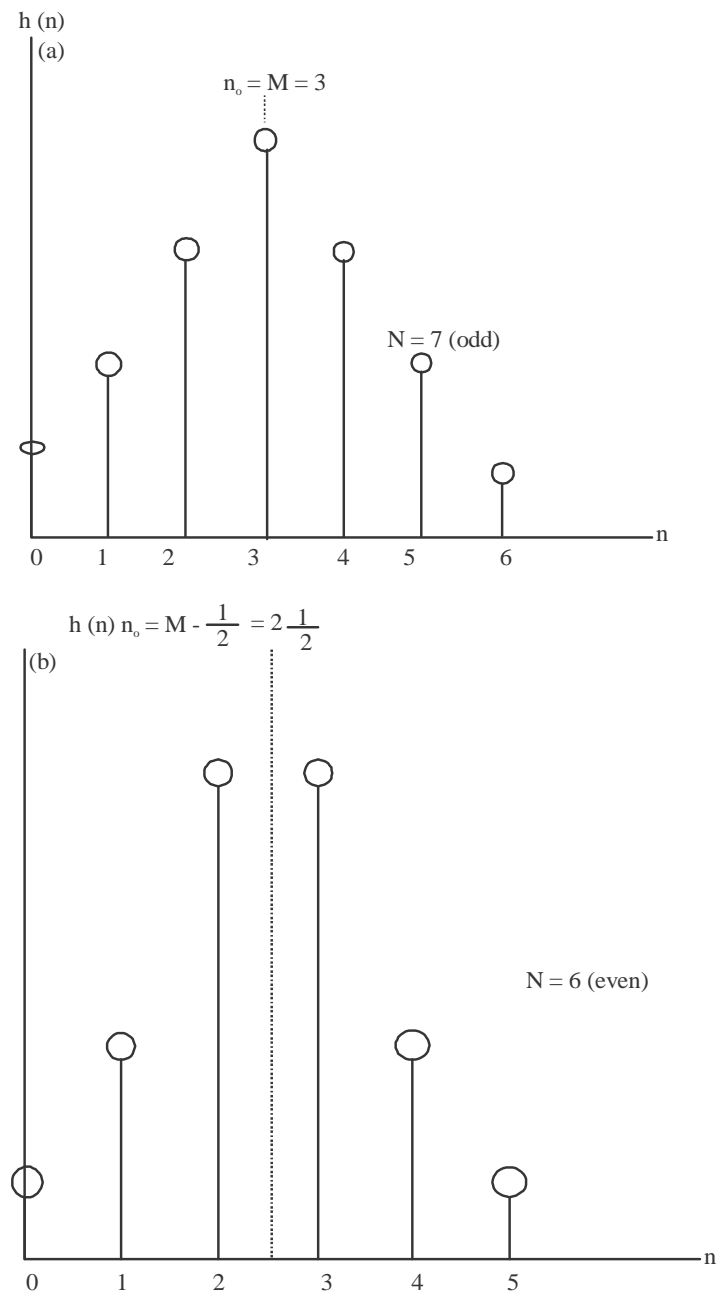

Fig. 8: (a) Symmetric impulse responses for an FIR filter and (b) Left: odd length. Right: even length

by the relation $\mathrm{h}(\mathrm{n})=\mathrm{h}(\mathrm{N}-\mathrm{n}-1)$ or anti-symmetric around $\mathrm{n}=0$, hence, $-\mathrm{h}(\mathrm{n})=\mathrm{h}(\mathrm{N}-\mathrm{n}-1))$. If $\mathrm{N}$ is an odd integer, i.e., $\mathrm{N}=2 \mathrm{M}+1$ the midpoint of symmetry is $\mathrm{n}_{0}=\mathrm{M}$. If $\mathrm{N}$ is an even integer, i.e, $\mathrm{N}=2 \mathrm{M}$ the midpoint of symmetry is $\mathrm{n}_{0}=\mathrm{M}-1 / 2$ (Fig. 8).

The Signal-to-Noise Ratio (SNR) is defined for analog signals as the ratio of the signals as the ratio of the signal power $\mathrm{p}_{\mathrm{x}}$ to the noise power $\mathrm{p}_{\mathrm{n}}$, i.e, $\mathrm{SNR}=\mathrm{r}=\mathrm{p}_{\mathrm{x}} / \mathrm{p}_{\mathrm{n}}$. Normally we use the logarithm value in $\mathrm{dB}$ :

$$
\mathrm{SNR}_{\mathrm{dB}}=10 \log _{10}\left(\frac{\mathrm{p}_{\mathrm{x}}}{\mathrm{p}_{\mathrm{n}}}\right)
$$

where, $\log _{10}$ is the common logarithm (base 10) (Hussain et al., 2011). However, we should note that when a random signal enters a system $H(f)$, the output signal would also be random whose Power Spectral Density (PSD) equals the input PSD multiplied by the power transfer function of the system which is the square of the transfer function $|\mathrm{H}(\mathrm{f})|^{2}$. Now assume white gaussian noise input $\mathrm{n}(\mathrm{t})$ with constant PSD entering an FIR filter. The output color noise PSD is given by:

$$
\mathrm{SNR}_{\mathrm{dB}}=10 \log _{10}\left(\frac{\mathrm{p}_{\mathrm{x}}}{\mathrm{p}_{\mathrm{n}}}\right)
$$

\section{RESULTS AND DISCUSSION}

We can measure the performance of the CSK system generated using the LCG1 map in different noisy environments with different SFs. The following results show the BER for SNRs ranging from [-20, 20]. The BER is used to measure the effect of channel imperfections on the transmitted signal that is obtained by counting the number of incorrectly received bits and dividing this number by the total number of bits transmitted. Simulation results of comparing and measuring the performance of CSK and DCSK systems under Gaussian noise and color noise to determine in which case the system is more secure and resistant of noise in channel communications.

We will compare simulation results obtained for different values of SF $=(4,6,10), n(F I R)=(0,6,20)$ and tow type of Chaos maps (Tent, Logistic) with SNR $=[-0,20]$. Firstly we similate DCSK and CSK under Additive White Gaussian Noise AWGN and Color Noise CN for different Spreading Factor (SF) values and n (FIR) length $=10$ with Logistic map and Tent map.

The obtained results in Fig. 9 we can see that when CSK is in AWGN environment and has its spreading factor increased, a lower bit error rate is obtained resulting in a decrease in the effective noise on the signal that was sent. Conversely, when the SF is set to a smaller value, the BER for a given SNR is increased. We can compare the performance of CSK using the LCG1 map with another Chaos generator (such as the tent map) in Fig. 10 in AWGN environment. When the SF is set to a smaller value, the BER for a given SNR is increased same when using LCGI map but the logistic map given the best performance (i.e., less error), Fig. 9 and the comparison in Fig. 11.

In Fig. 12 we can see that when DCSK is in AWGN environment and has its spreading factor decreased, a lower bit error rate is obtained resulting in a decrease in the effective noise on the signal that was sent. When the SF is set to a smaller value, the BER for a given SNR is decreased, small SF give small BER at high SNR.

We can compare the performance of DCSK using the LCG1 map with another Chaos generator (Tent map), Fig. 13. We can conclude from this that LCGI map has better performance than the tent map, especially at high SNRs and low SF where it gives less error (Fig. 14).

From Fig. 15 DCSK vs. CSK with the logistic map under Gaussian noise: DCSK performance is better than CSK under Gaussian noise also DCSK is more popular, 


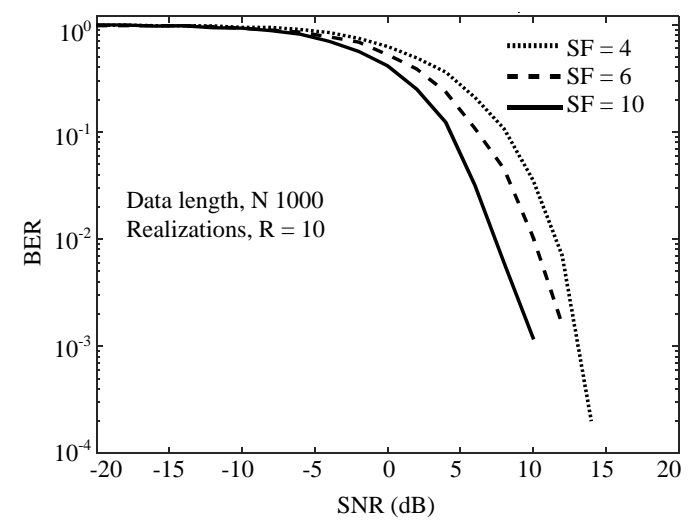

Fig. 9: CSK BER vs. SNR using the logistic map under Gaussian noise; (CSK BER vs. SNR, noise = Gaussian map = LG)

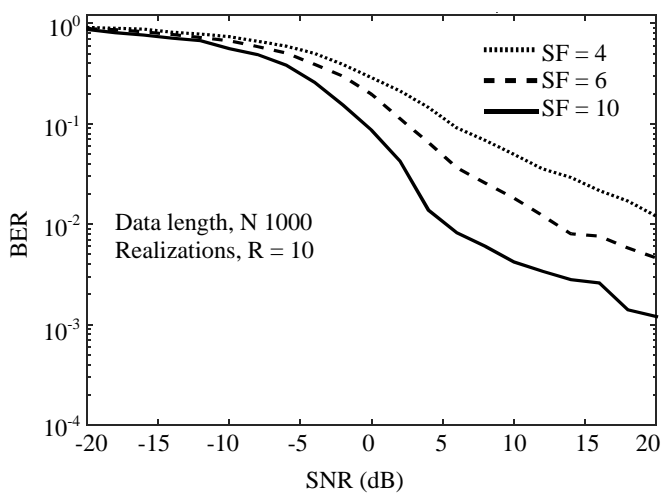

Fig. 10: CSK BER vs. SNR using map tent under Gaussian noise; (CSK BER vs. SNR, noise = Gaussian map $=$ tent $)$

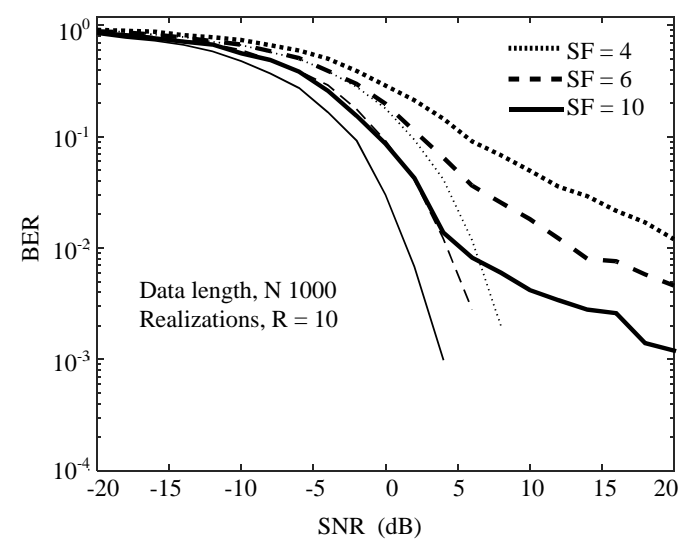

Fig. 11: CSK BER vs. SNR using tent and the LG map under Gaussian noise; (CSK BER vs. SNR, noise $=$ Gaussian, map $=$ tent (thick) and LG)

since, it is simpler in implementation. In Fig. 15, we noted a small error when using a DCSK system with logistic

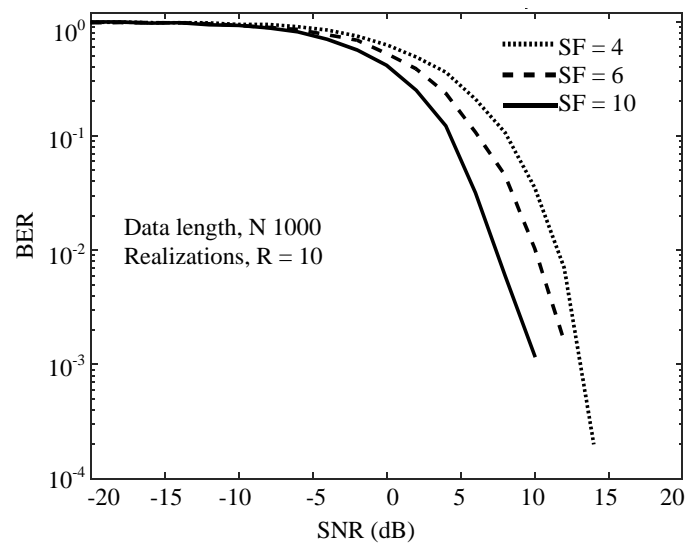

Fig. 12: DCSK BER vs. SNR using the logistic map under Gaussian noise; (DSCK BER vs. SNR, noise $=$ Gaussian map $=$ LG)

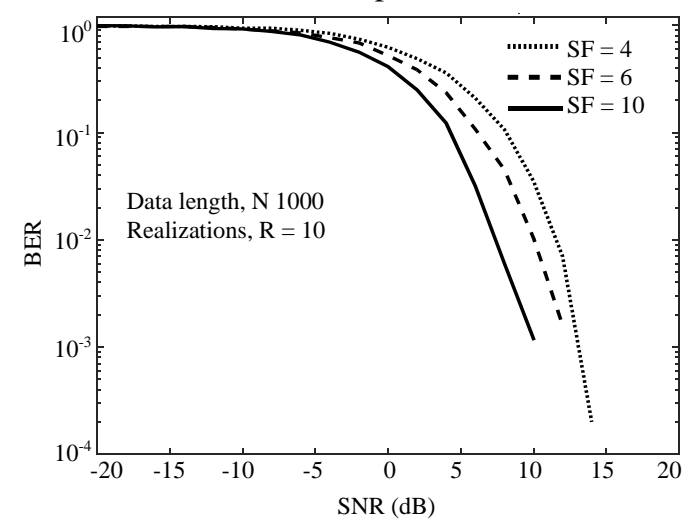

Fig.13: DCSK BER vs. SNR using tent map under Gaussian noise; (DSCK BER vs. SNR, noise = Gaussian, map = tent)

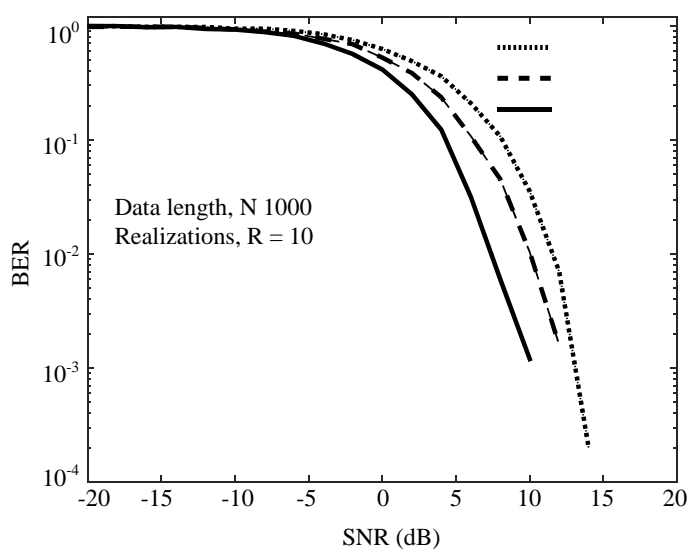

Fig.14: DCSK BER VS. SNR using tent and LG under Gaussian noise; (DSCK BER vs. SNR, noise = Gaussian map = tent (thick and LG))

map with the highest value of SNR and less value of $\mathrm{SF}\left(\mathrm{SF}=4, \mathrm{SNR} \cong 15, \mathrm{BER} \cong 10^{-4} \mathrm{~dB}\right)$. DCSK and CSK 


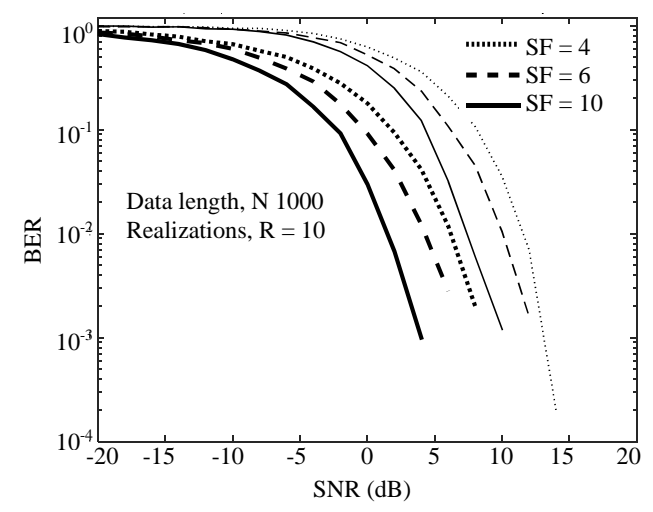

Fig.15: DCSK and CSK BER v's. SNR using logistic mapunder Gaussian noise; (CSK (thick and DCSK BER vs. SNR, noise = Gaussian map = LG))

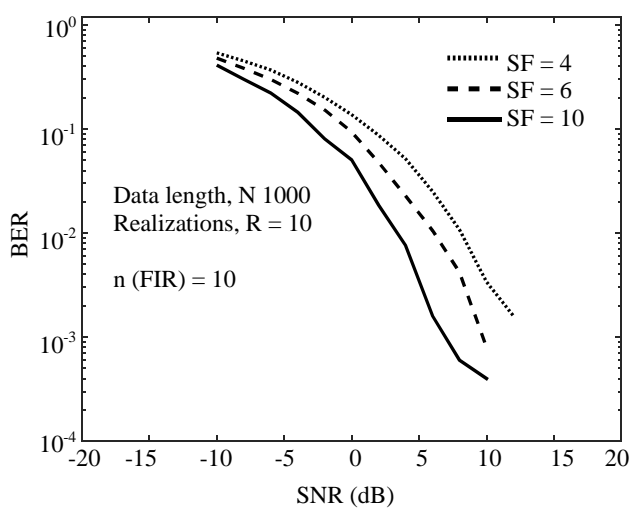

Fig. 16: CSK BER vs. SNR using logistic map under color noise; (CSK BER vs. SNR noise = color map = LG)

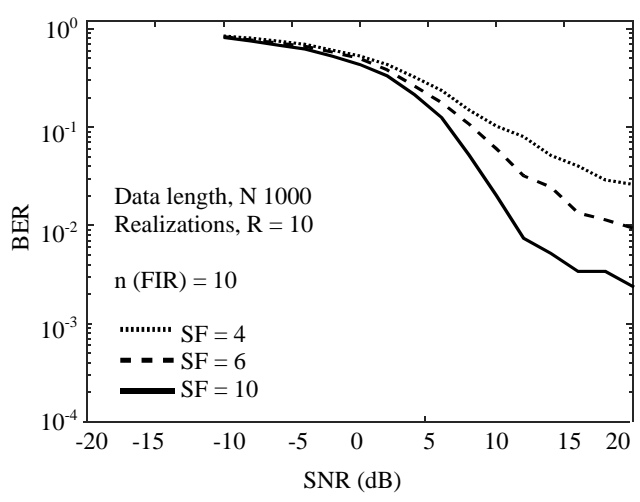

Fig. 17: CSK BER vs. SNR using tent map under color noise; (CSK BER vs. SNR, noise = color map = tent)

under color noise with FIR order $n=10$ using logistic map and tent map and different values of spreading factor.

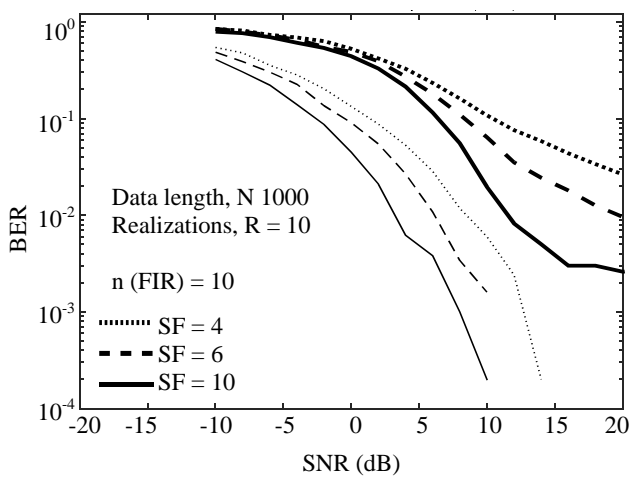

Fig. 18: SK BER vs. SNR using tent and logistic under colornoise; (CSK BER vs. SNR noise = color map tent (thick) and LG))

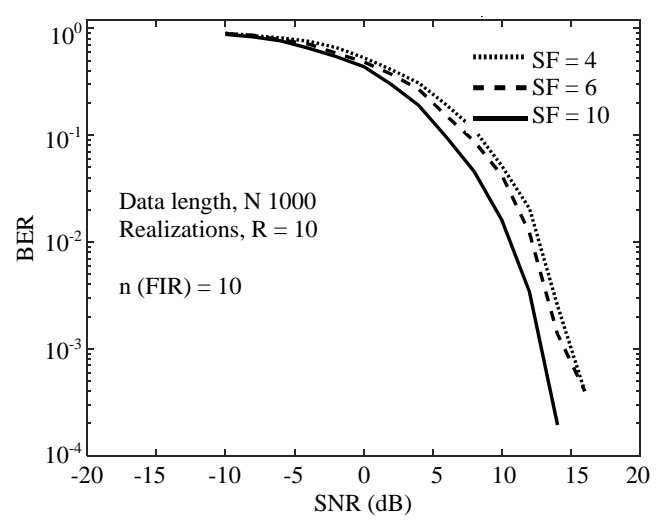

Fig.19: DCSK BER vs. SNR using logistic map under color noise; (DCSK BER vs. SNR, noise = color map = LG)

In Fig. 16, we can see that when CSK is in CGN (color white Gaussian noise) environment and under the logistic map has best performance when the SF is set to a bigger value, the BER for a given SNR is decreased, best values of our factors ( $S F=10$, FIR length $=10$, SNR a lower bit error rate $\mathrm{BER} \cong 10^{-4}$ is obtained resulting in a decrease in the effective noise on the signal that was sent.

In Fig. 17, we can see that when CSK is in CWGN (color noise) environment and under the tent map has best performance when the SF is set to a bigger value, the BER for a given SNR is decreased, best values of our factors $(S F=10$, FIR length $=10, S N R \geq 20)$ a lower bit error rate $10^{-3}<\mathrm{BER}<10^{-2}$ is obtained resulting in a decrease in the effective noise on the signal that was sent. In Fig. 18, a comparison between logistic map and Tent map with CSK system is made, we can see that when CSK is under CWGN (color noise) environment with logistic map has better performance.

In Fig. 19, we can see that when DCSK is under CWGN environment and using logistic map has best performance when the SF is set to a bigger value, the 


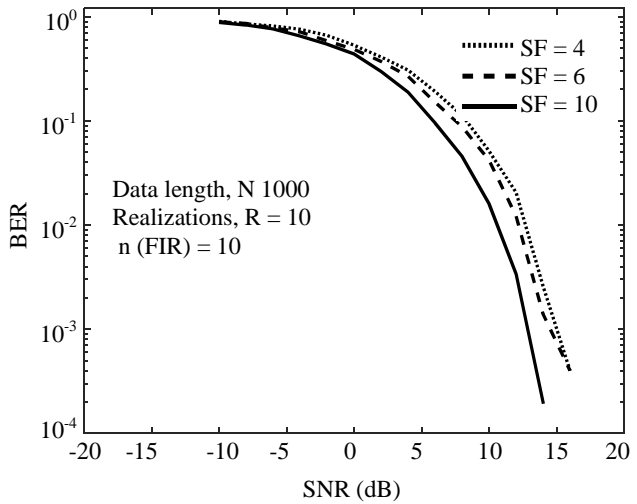

Fig. 20: DCSK BER vs. SNR using tent map under color noise; $($ DCSK BER vs. SNR, noise = color map $=$ tent)

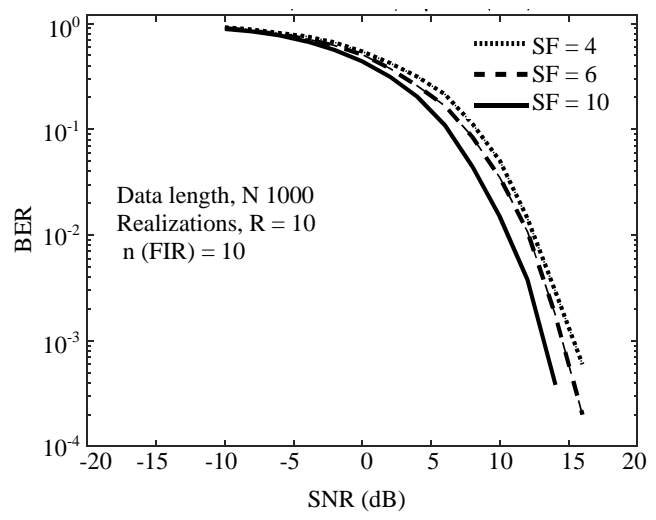

Fig. 21: DCSK BER vs. SNR using tent and LG under color Gaussia noise; (DCSK BER vs. SNR, noise $=$ color map $=$ tent (thick) and LG)

BER for a given SNR is decreased, best values of our factors (SF $=10$, FIR length $=10, \mathrm{SNR} \cong 15$ ) a lower bit error rate $\mathrm{BER} \cong 10^{-4}$.

In Fig. 20 we can see that when DCSK is under CN environment using tent map has best performance when the SF is set to a bigger value, the BER for a given SNR is decreased, best values of our factors ( $\mathrm{SF}=10$, FIR length $=10, \mathrm{SNR} \cong 15$ a lower bit error rate $\mathrm{BER} \cong 10^{-4}$. In Fig. 21 comparison between logistic map and tent map with DCSK system, we can see that when DCSK is in CN (color noise) environment and under the logistic map has better performance. Figure 22 shows the difference between CSK and DCSK under color noise in logistic map environment as a result the CSK has best performance.

Compare Fig. 15 (Gaussian) with Fig. 22 (color) our systems under logistic map for both CSK and DCSK, color noise is more damaging than Gaussian (gives more error at any specific SNR). CSK has better performance than DCSK in AWGN and CN.

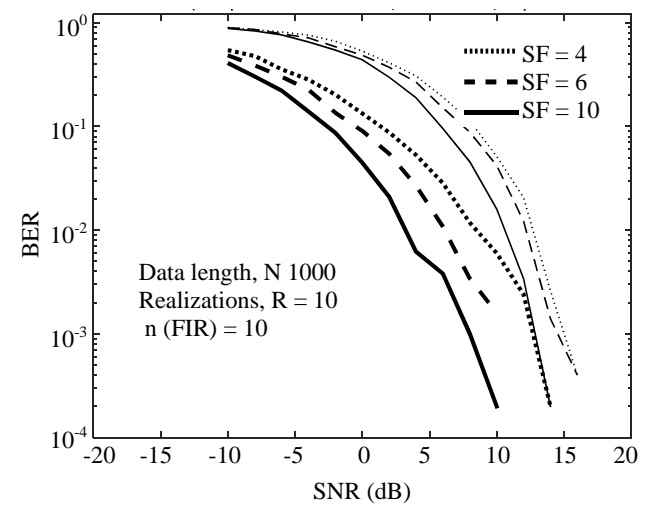

Fig. 22: DCSK and CSK BER vs. SNR using logistic map under color noise; (CSK (thick) and DCSK BER vs. SNR, noise = color map $=\mathrm{LG}$ )

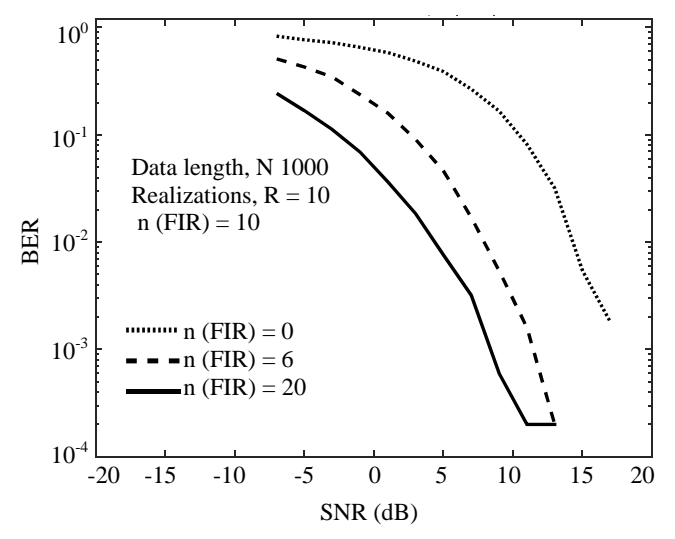

Fig. 23: CSK with different lengths of FIR under the logistic map (CSK BER vs. SNR, different $n$ $($ FIR) map $=$ LG)

We now simulate DCSK and CSK under color additive noise $C N$ with different Filter length $n(F I R)=(0,6,20)$ and spreading factor $(\mathrm{SF}=10)$ with Logistic map and tent map. The obtained results in Fig. 23, we can see that when CSK has a lower bit error rate is obtained resulting in a decrease in the effective noise on the signal that was sent. When the filter length set to a bigger value, the $\mathrm{BER}$ for a given $\mathrm{SNR}$ is decreased, $\mathrm{n}(\mathrm{FIR})=20, \mathrm{BER} \cong$ $10^{-4}$ SNR $>10$.

We can compare the performance of CSK using the LCG1 map with another Chaos generator (such as the tent map) in Fig. 24 when the n (FIR) is set to 10 value, $\mathrm{SNR} \geq 20$ the $10^{-3}<10^{-2} \mathrm{BER}$ for a given SNR is decreased same when using LCG1 map but the logistic map given the best performance (i.e., less error) (Fig. 25).

Figure 26-29, we can see that we have less error when using a DCSK system under the logistic map which has better performance than tent, especially at high SNR 


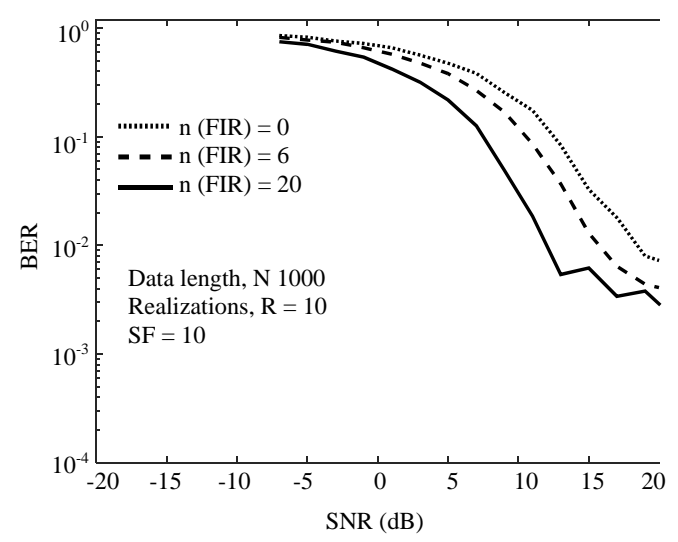

Fig. 24: CSK with different lengths of FIR under tent map (CSK BER vs. SNR, different $n$ (FIR), map $=$ tent)

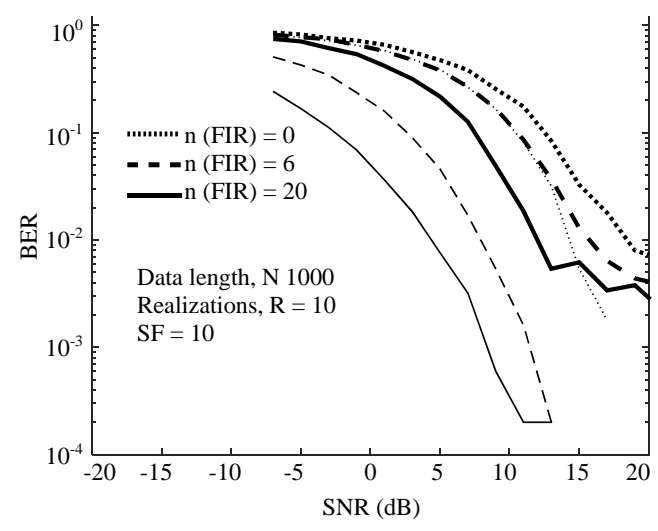

Fig. 25: CSK with different lengths of FIR under tent map and logistic map (CSK BER vs. SNR, different $n$ (FIR), map = tent (thick and LG))

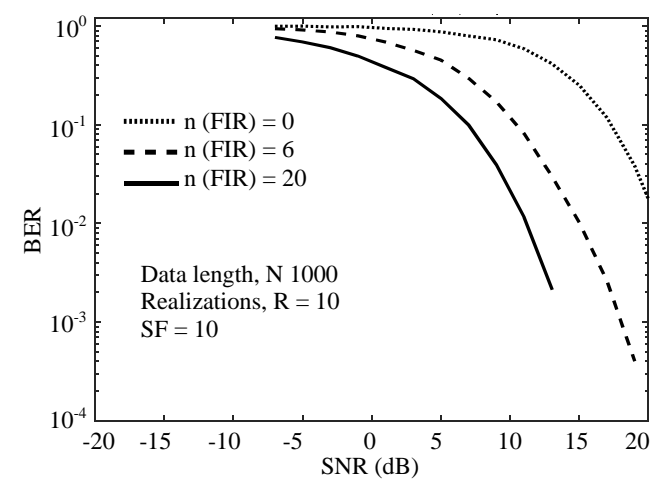

Fig. 26: DCSK with different lengths of FIR under the logistic map (DCSK BER vs. SNR, different $n$ $(\mathrm{FIR})$, map = LG)

where it gives less error. At high SNR, the difference grows much bigger. Best result when $\mathrm{n} \quad(\mathrm{FIR})=6, \quad \mathrm{SNR}>20$ that gives $10^{-4}<\mathrm{BER}<10^{-3}$

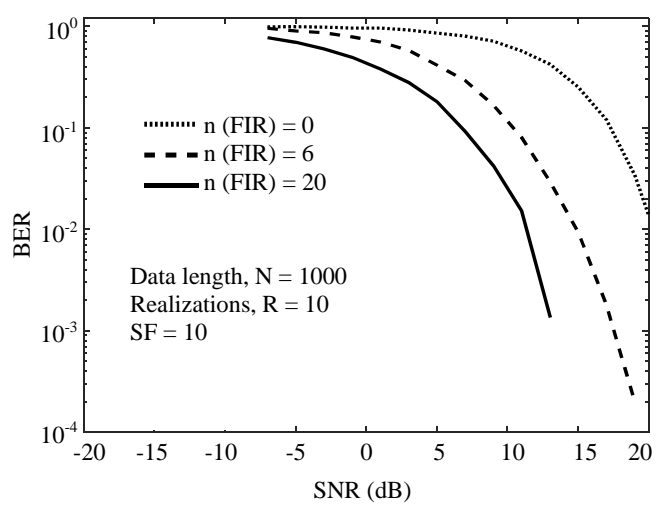

Fig. 27: DCSK with different lengths of FIR under the tent map (DCSK BER vs. SNR, different $\mathrm{n}$ (FIR), map = tent)

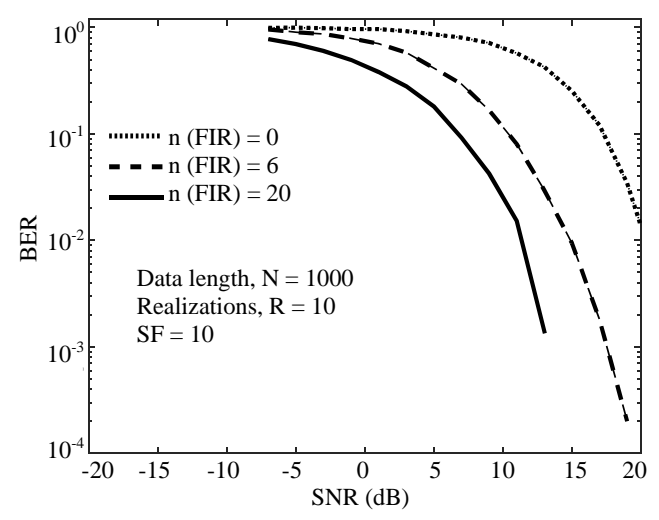

Fig. 28: DCSK with different lengths of FIR under tent map and logistic map. Zero length means no filter (DCSK BER vs. SNR, different n (FIR), map = tent (thick) and LG)

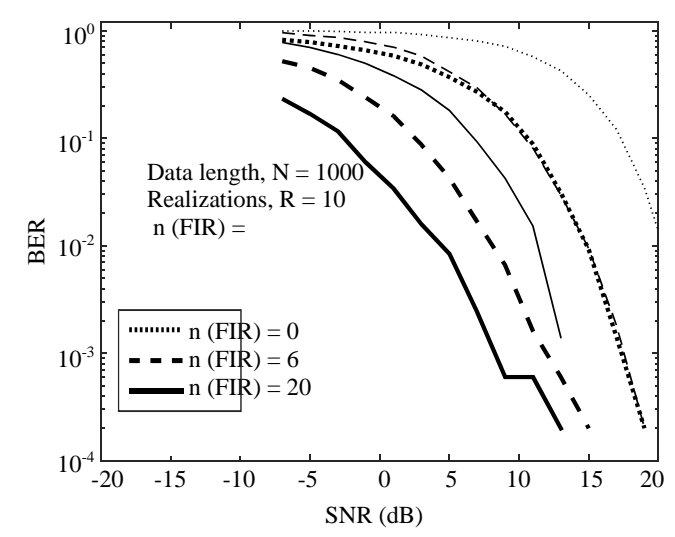

Fig. 29: CSK and DCSK with different lengths of FIR under the logistic map, CSK outperforms DCSK in BER performance (reduced error) (CSK (thick) and DCSK BER vs. SNR, different $n$ (FIR), map = LG) 
Note: From Fig. 28 and 29, we can that larger length of the filter gives more error because it allows passing more noise. Further research could be performed to find the performance under modern communication systems as by Lau and Hussain (2005), Mahmoud et al. (2006).

\section{CONCLUSION}

In this research generated color noise to test the perfroamnce of Chaos Shift Keying (CSK) and Differential Chaos Shift Keying (DCSK) systems as compared to their performance under Gaussian noise. The chaotic maps of logistic and tent maps have been used in building the two systems with different values of Spreading Factor (SF). Color noise has been generated using FIR filtering of Gaussain noise where different filter orders n (FIR) have been used in this study. As expected, larger length of the filter gives more error as it allows the passing of more noise. It is shown that CSK outperforms DCSK under additive white Gaussian noise (lower error by nearly 10dB) also CSK has better security but DCSK is more popular, since, it is simpler in implementation.

\section{ACKNOWLEDGEMENT}

The researchers would like to thank the Ministry of Higher Education and Scientific Research in Iraq for financial support of this project.

\section{REFERENCES}

Abel, A. and W. Schwarz, 2002. Chaos communications-principles, schemes and system analysis. Proc. IEEE., 90: 691-710.

Cai, G., Y. Fang, G. Han, L. Wang and G. Chen, 2017. A new hierarchical \$M\$-ary DCSK communication system: Design and analysis. IEEE. Access, 5: 17414-17424.

Dedieu H., M.P. Kennedy and M. Hasler, 1993. Chaos shift keying: Modulation and demodulation of a chaotic carrier using self-synchronizing Chua's circuits. Trans. Circ. Sys., 40: 634-642.

Dedieu, H. and M.J. Ogorzalek, 1997. Identifiability and identification of chaotic systems based on adaptive synchronization. IEEE. Trans. Circuits Syst. I. Fundam. Theor. Appl., 44: 948-962.

Elmirghani, J.M.H., 1995. Data communication via. chaotic encoding and associated security issues. Proceedings of the International Conference on Global Telecommunications (GLOBECOM'95), November 14-16, 1995, IEEE, Singapore, pp: 1188-1192.

Gleick, J., 1987. Chaos: Making a New Science. Viking Press, ?New York, USA., ISBN:9780670811786, Pages: 352.
Hogg, R.V. and A.T. Craig, 1978. Introduction to Mathematical Statistics. 4th Edn., Macmillan Publishing Company, New York.

Hussain Z.M., A.Z. Sadik and P. O’Shea, 2011. Digital Signal Processing: An Introduction with MATLAB and Applications. Springer, Berlin, Germany, ISBN:978-3-642-15590-1, Pages: 350.

Jawad, S.T.M., Z.M. Hussain and K. Neville, 2017. A study on the performance of CSK under noisy conditions. Intl. J. Appl. Eng. Res., 12: 11840-11846.

Kaddoum, G., 2016. Wireless Chaos-based communication systems: A comprehensive survey. IEEE. Access, 4: 2621-2648.

Kolumban, G., 2000. Theoretical noise performance of correlator-based chaotic communications schemes. IEEE. Trans. Circuits Syst. I. Fundam. Theor. Appl., 47: 1692-1701.

Kolumban, G., B. Vizvari, W. Schwarz and A. Abel, 1996. Differential Chaos shift keying: A robust coding for Chaos communication. Proceedings of the International Workshop on Nonlinear Dynamics of Electronic Systems, June 27-28, 1996, Seville, Spain, pp: 87-92.

Kolumban, G., M.P. Kennedy, Z. Jako and G. Kis, 2002. Chaotic communications with correlator receivers: Theory and performance limits. Proc. IEEE., 90: 711-732.

Larson, L.E., J.M. Liu and L.S. Tsimring, 2006. Digital Communications using Chaos and Nonlinear Dynamics. Springer, Berlin, Germany, ISBN-13:978-0387-29787-3, Pages: 379.

Lau, F.C.M. and C.K. Tse, 2003. Co-existence of Chaos-based and conventional digital communication systems. Proceedings of the 2003 International Symposium on Circuits and Systems (ISCAS'030), May 25-28, 2003, IEEE, Bangkok, Thailand, pp: 204-207.

Lau, F.C.M., C.K. Tse, M. Ye and S.F. Hau, 2004. Coexistence of Chaos-based and conventional digital communication systems of equal bit rate. IEEE. Trans. Circuits Syst I. Regul. Pap., 51: 391-408.

Lau, Y., 2006. Techniques in secure Chaos communication. Ph.D Thesis, RMIT University, Melbourne, Australia.

Lau, Y.S. and Z.M. Hussain, 2005. A new approach in choas shift keying for secure communication. Proceedings of the 3rd IEEE International Conference on Information Technology and Applications Vol. 2, July 4-7, 2005, IEEE, Sydney, New South Wales, ISBN:0-7695-2316-1, pp: 630-633.

Lau, Y.S., K.H. Lin and Z.M. Hussain, 2005. Space-time encoded secure Chaos communications with transmit beam forming. Proceedings of the 2005 IEEE International Conference on Region 10 (TENCON2005), November 21-24, 2005, IEEE, Melbourne, Queensland, Australia, pp: 1-5. 
Lian, K.Y., T.S. Chiang, C.S. Chiu and P. Liu, 2001. Synthesis of fuzzy model-based designs to synchronization and secure communications for chaotic systems. IEEE. Trans. Syst. Man Cybern. Part. B., 31: 66-83.

Luke, O.D., 2012. Chaos based secure communication and system design. MSc Thesis, Tshwane University of Technology, Pretoria, South Africa.

Mahmoud, S.S., Z.M. Hussain and P. O'shea, 2006. A geometrical-based microcell mobile radio channel model. Wireless Networks, 12: 653-664.

Rao, S.S. and S.P. Howard, 1996. Correlation performance of chaotic signals in spread spectrum systems. Proceedings of the 1996 IEEE International Workshop on Digital Signal Processing, September 1-4, 1996, IEEE, Loen, Norway, pp: 506-509.
Rupak., K., 2011. Design and implementation of secure chaotic communication systems. Ph.D Thesis, Northumbria University, Newcastle Upon, England, UK.

Strogatz, S.H., 1994. Nonlinear Dynamics and Chaos: With Applications to Physics, Biology, Chemistry and Engineering. Perseus Books Group, New York, USA.,. Sun, K., 2016. Chaotic Secure Communication: Principles and Technologies. Walter de Gruyter, Berlin, Germany, ISBN-13:978-3110426885, Pages: 348.

Ushio, T., 1994. Control of chaotic synchronization and secure communication systems. Proceedings of the 1994 IEEE International Symposium on Emerging Technologies and Factory Automation (ETFA'94), November 6-10, 1994, IEEE, Tokyo, Japan, pp: 231-238. 